\title{
Analysis of Material Removal and Surface Characteristics in Machining Multi Walled Carbon Nanotubes Filled Alumina Composites by WEDM Process
}

\author{
Meinam Annebushan Singh ${ }^{1}$, Deba Kumar Sarma ${ }^{2 a}$, Hriday Mani Kalita ${ }^{3}$ and Comingstarful Marthong ${ }^{4}$ \\ ${ }^{1}$ NIT Meghalaya, Mechanical and Civil Engineering Department, 793003 Shillong, India
}

\begin{abstract}
The reinforcement of ceramic materials with electrically conductive particles increases the overall conductivity of the ceramic material. This allows the ceramic material to be more readily machined using wire electrical discharge machining process. The current work is an approach to identify the machinability of multi walled carbon nanotubes filled alumina composites in wire electrical discharge machining process. Alumina samples of 5 vol. \% and 10 vol. \% multi walled carbon nanotubes are machined and analysed for material removal rate and the surface characteristics. An increase in material removal rate is observed with increase in filler concentrations. At the same time, better surface roughness is observed. The surface characteristics of composite alumina are further compared with Monel 400 alloy. It has been observed that spalling action is the dominating material removal mechanism for alumina composites, while melting and evaporation is for the Monel 400 alloy.
\end{abstract}

\section{Introduction}

Electrical Discharge Machining (EDM) and Wire Electrical Discharge Machining (WEDM) is a well-known non-conventional machining process for machining conductive materials. A wide range of materials can be successfully machined in this method by using a relatively softer tool material as long as they are conductive in nature. The machining phenomenon has been made successful as there is no relative contact between the tool and the work piece. In the normal machining scenario, the tool is made the cathode and the work piece material as anode. Both the tool and work piece are separated by a very small distance, known as the spark gap. The gap is then filled with a dielectric medium, generally hydrocarbon based oil in case of EDM, while deionised water for the case of WEDM. When current is supplied in the system, a potential difference is generated in the system. Due to the generated potential difference, the dissociation of the dielectric fluid occurs, which allows the transfer of electrons from cathode to anode. This leads to an intense generation of sparks from tool to the workpiece. The striking of sparks causes an intense localised heating that leads to melting of the work piece at that specific location. Here, most of the melted materials are either evaporated or flushed away under the action of the dielectric fluid. However, some portion of the work piece material gets re solidified and settles down on the surface as heat affected zone, commonly known as the recast layer.

A major challenge till date is the possibility to machine ceramic and its composites in EDM/WEDM process. It is a wellknown fact that as the overall electrical conductivity of the ceramic material is very low. This restricts the usage of EDM/WEDM process to machine ceramic materials. However, researchers have identified two alternate approaches to machine ceramic in EDM/WEDM process. The first approach is the inclusion of a conductive layer, known as the Assisting Electrode (AE) on the surface of the electrically non-conductive ceramic [1]. The applied AE layer being conductive in nature helps in initial triggering of sparks. After the completion the AE layer, further machining is carried forward by the continuously generated pyrolytic carbon particles. The carbon particles are continuously generated due to the breaking and dissociation of kerosene (hydrocarbon based dielectric fluid). A continuous formation of the carbon layer on the surface of the work piece ensures further machining to propagate. Thus, dependence on hydrocarbon based dielectric oil is a must to sustain further machining. Various types of AE material were reported by various researchers that can successfully trigger the initial machining process [2-4]. Further, researchers have modified the general WEDM setup to a die sinking type WEDM to facilitate the use of kerosene [5-6]. A successful machining was reported in this regard. In the current approach, the overall material removal rate was found to be very low and the obtained surface finish was very rough.

Another approach that researchers adopted to machine ceramics in EDM/WEDM process is by reinforcing the ceramic material with conductive filler. This method increases the overall electrical conductivity of the ceramic material, thereby rending it to be machinable in EDM/WEDM [7-11]. Researchers have used TiN, TiC, TiCN, $\mathrm{SiC}, \mathrm{NbC}, \mathrm{TiB}_{2}$ and $\mathrm{WC}$ as the reinforcing materials. An increase in the overall electrical conductivity of ceramic materials like $\mathrm{Al}_{2} \mathrm{O}_{3}, \mathrm{ZrO}_{2}$ and $\mathrm{Si}_{3} \mathrm{~N}_{4}$ were observed. This allowed machining of ceramic material with a much lesser ease. However, the percentage volume incorporation of such conducting fillers were found to be very high (approximately $30 \mathrm{vol}$. \%). This alters the overall properties of the ceramic material as a whole. An improvement in this method is the use of carbon nanotubes (CNT) as the reinforcing conductive fillers. A small amount of the CNT particle can considerably increase the overall electrical conductivity of the composites [12]. Thus a good

\footnotetext{
${ }^{a}$ Corresponding author : dksarma@nitm.ac.in
} 
machining aspect has been obtained with CNT as the conductive filler. Further, as a strengthening means of this approach, Melk et al [13] reported that the use of multi walled carbon nanotubes (MWCNT) in zirconia ceramics at very low concentrations facilitate effective machining capabilities in EDM process. Singh et al [14] made an initial effort to identify the machining aspect of MWCNT filled alumina composites in WEDM process. Substantial machining capabilities were observed with 7.5 vol \% MWCNTs or more. Keeping the above reported works in mind, the researchers have decided to further analyse the machinability aspect of MWCNT filled alumina composites. The variation of the material removal rate and the surface characteristics are identified and discussed in this paper. To get a better understanding of the surface characteristics, a comparison of the machining of alumina composites and Monel 400 alloy is represented.

\section{Experimental Works}

The main aim of this work is to identify the machinability aspect of MWCNT filled alumina composites in WEDM process and then to further compare it with Monel 400 alloy. Two samples of MWCNT concentration of 5 and $10 \%$ by volume are considered [15]. It has been observed that the electrical conductivity is found to increase with the increase in CNTs, while the thermal conductivity decreases correspondingly (Table 1). The increase in electrical conductivity is put forward by the increasing concentrations of MWCNTs that increases the overall electrical network in the composites. On the other hand, decrease in thermal conductivity is attributed by the porosity of the composites and the grain boundary interactions. Anisotropy properties are observed in the samples due to random orientations of the CNTs. WEDM setup (DK7732, Concord Limited, Bangalore, India) has been used to perform the machining process (Fig. 1). The various input parameter used for the current machining process is represented in table 2. Further, the surface roughness of the machined samples are analysed using a surface roughness tester (Surtronic S-128, Taylor Hobson). A scanning electron microscope (SEM; JSM - 6360 JEOL) is then used to analyse the surface and the sub surface characteristics.

Table 1. Properties of MWCNT filled alumina composites

\begin{tabular}{|c|c|c|c|}
\hline MWCNT & Density $\left(\mathbf{k g} / \mathbf{m m}^{\mathbf{3}}\right)$ & Electrical Conductivity $(\mathbf{S} / \mathbf{m})$ & Thermal Conductivity $(\mathbf{W} / \mathbf{m} \cdot \mathbf{K})$ \\
\hline 5 vol. $\%$ & 3840.80 & 479 & 24.72 \\
\hline 10 vol. $\%$ & 3672.20 & 1402.50 & 15.38 \\
\hline
\end{tabular}

Figure 1. WEDM setup.

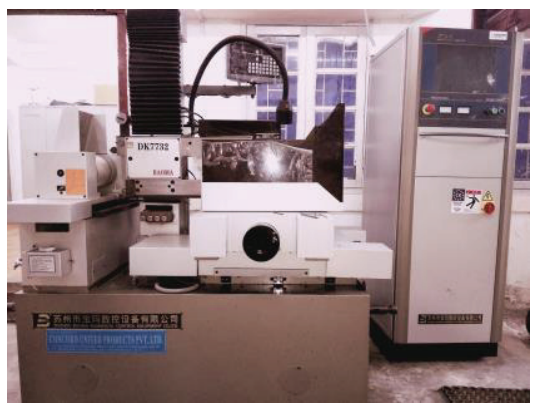

Table 2. Input parametric conditions

\begin{tabular}{|c|c|}
\hline Parameters & Values \\
\hline Pulse on time $\left(\mathrm{T}_{\text {on }}\right)$ & $25 \mu \mathrm{s}$ \\
\hline Pulse off time $\left(\mathrm{T}_{\text {off }}\right)$ & $9 \mu \mathrm{s}$ \\
\hline Wire speed setting & $0(1493 \mathrm{rpm})$ \\
\hline Current & $2 \mathrm{~A}$ \\
\hline $\begin{array}{c}\text { Dielectric medium \& } \\
\text { conductivity }\end{array}$ & $\begin{array}{c}\text { Deionised water \& } \\
0.7 \mu \mathrm{S} / \mathrm{cm}\end{array}$ \\
\hline $\begin{array}{c}\text { Wire material \& } \\
\text { Diameter }\end{array}$ & $\begin{array}{c}\text { Molybdenum \& } \\
0.18 \mathrm{~mm}\end{array}$ \\
\hline
\end{tabular}

\section{Results and Discussion}


The experiments are performed on two types of MWCNT alumina composites and in Monel 400 alloy with the same input parametric conditions as shown in table 2. The experiments are performed with the material removal rate (MRR) and surface roughness $\left(\mathrm{R}_{\mathrm{a}}\right)$ as the output performance characteristics. The experimental results are shown in table 3 .

It has been found that the MRR increases considerably with increase in MWCNT concentration (Fig. 2). The increase in MRR has been brought forward by the incorporation of higher CNTs in the composites. With a comparatively higher CNTs in the composite, the electrical network increases, thereby increasing the overall passage of current. Thus, this condition ensures an increase in the net heat flux generated in between the tool and the alumina composites. Though the MRR is increasing for MWCNT alumina composite, it is not as prominent as that of Monel 400. The vast difference in MRR at a particular parametric setting is due to the immense difference in the electrical conductivity of the materials (Fig. 2). The Monel alloy being more conductive, have a higher tendency to allow the current to pass through. Thus, a higher material removal phenomenon is observed.

Table 3. Experimental results

\begin{tabular}{|c|c|c|}
\hline Material Types & $\begin{array}{c}\text { Material removal rate } \\
\left(\mathbf{m m}^{3} / \mathbf{m i n}\right)\end{array}$ & $\begin{array}{c}\text { Surface Roughness } \\
(\boldsymbol{\mu m})\end{array}$ \\
\hline 5 vol. \% MWCNT alumina composite & 1.91 & 3.167 \\
\hline 10 vol. \% MWCNT alumina composite & 9.69 & 2.28 \\
\hline Monel 400 alloy & 16.27 & 3.56 \\
\hline
\end{tabular}

During machining of 5 vol. \% MWCNT filled alumina composites, some wire lag phenomena is observed. The condition arises as a consequence of the low conductivity of the material that restricts the effective machining capabilities. In some case, the sample breaks off before actual completion of the machining process due to wire lag phenomena. The wire lag is also expected during machining of 10 vol. \% MWCNT filled alumina composites. But, no appreciable wire lag was observed. This may be due to the overall increase in the electrical conductivity of the alumina composites with increase in MWCNT concentrations. On the other hand, wire lag phenomena is not observed in machining of Monel 400 alloy. This is due to the fact that Monel 400 alloy being highly conductive in nature; efficient machining capabilities can be obtained.

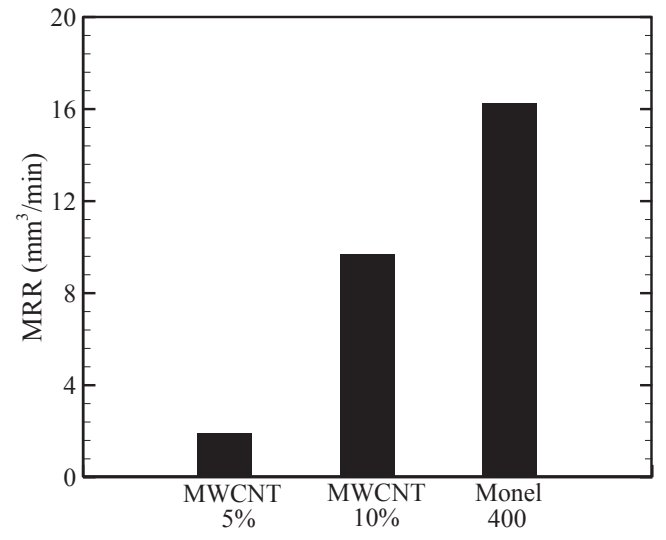

Figure 2. Variation of MRR of 5, 10 vol. \% MWCNTs alumina composites and Monel 400

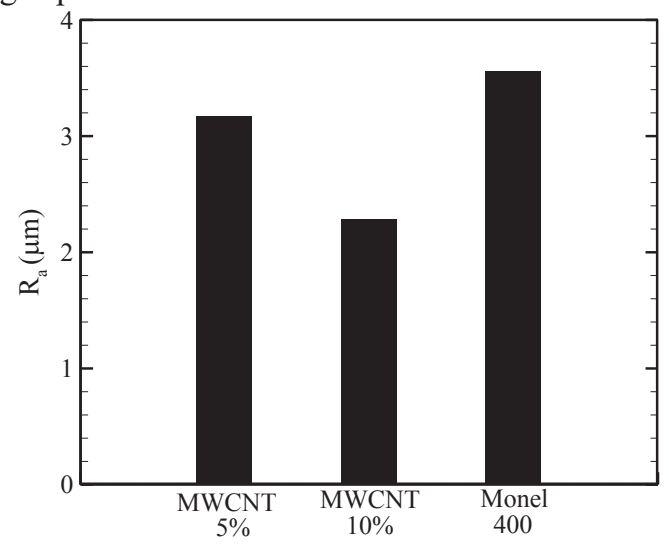

Figure 3. Variation of $\mathrm{R}_{\mathrm{a}}$ of 5,10 vol. \% MWCNTs alumina composites and Monel 400

Figure 3 shows the plot of surface roughness of the alumina composites and Monel 400 alloy. As expected, better $R_{a}$ is observed with increase in the MWCNT concentrations. During machining of conductive ceramic composites, the passage of electrical network is mainly due to the conductive reinforced fillers. Hence, the heating of reinforced fillers occurs initially. The intense generated heat is then dissipated to the neighbouring ceramic matrix materials. This leads to melting of the ceramic material by spalling action. Since the CNT concentration of 5 vol. \% MWCNT alumina composites is less, so the rate of heat transfer from the CNT to the alumina material is found to be lesser. Hence, the associated $\mathrm{R}_{\mathrm{a}}$ is found to be quite rough, coupled with a low MRR. However, for the case of 10 vol. \% MWCNT alumina composites, more alumina materials get heated due to the increasing CNT concentrations. Hence, a lower $\mathrm{R}_{\mathrm{a}}$ is obtained, coupled with a higher MRR. For the case of Monel 400 alloy, an intense localised heating occurs due to high electrical conductivity. At the same time, due to the inability to remove all the melted materials by the action of the cooling nature of the dielectric fluid, a portion of the melted material gets re solidified on the surface and forms a recast layer. The recast layer formation is generally associated with a high debris deposition on the surface. Therefore, a comparatively high $\mathrm{R}_{\mathrm{a}}$ value is observed in Monel 400 .

Figure 4 shows the SEM images to compare the surface characteristics of 5 vol. \%, 10 vol. \% MWCNT alumina composite along with Monel 400 alloy. A mixed spalling and melting phenomena is observed as the basic material removal characteristic in case of 5 and $10 \mathrm{vol}$ \% MWCNT alumina composites. However, for Monel 400 alloy, the melting and evaporation is seen as the material removal characteristics. For 5 vol. \% MWCNT alumina composite, the surface is highly porous in nature with ample amount of recast layer (Fig. 4a). However, for 10 vol. \% MWCNT alumina composite, the machined surface is found less porous in nature (Fig. 4b). The irregular debris particles on the machined surface are due to the effect of spalling mechanism while regular spherical debris due to the melting and evaporation effect. Thus, it is observed that spalling effect is more prominent with 10 vol. \% MWCNT alumina composites, while melting and evaporation is the dominant factor for Monel 400(Fig. 4c). 


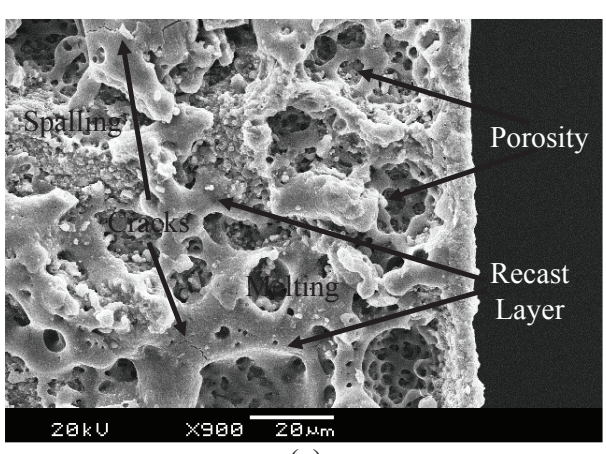

(a)

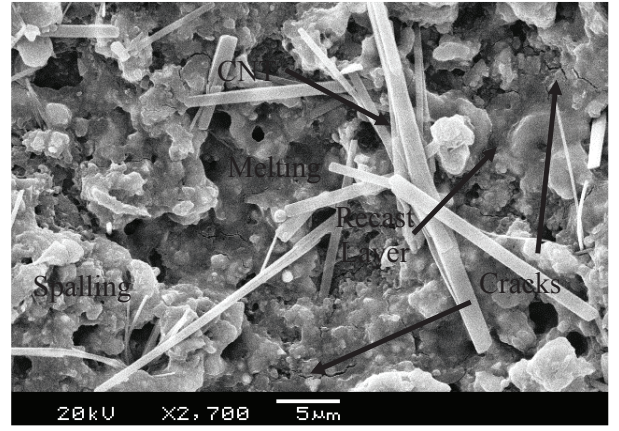

(b)

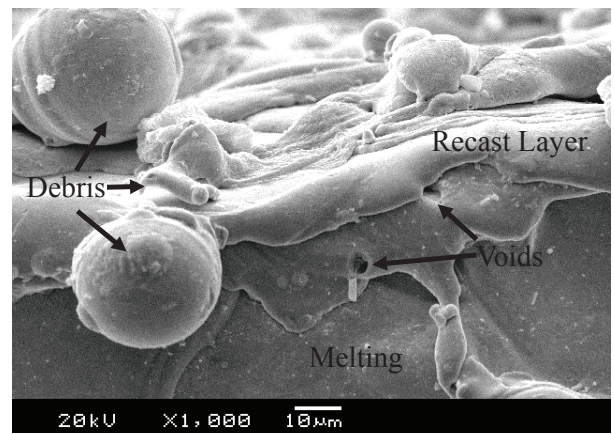

(c)

Figure 4. SEM images for surface characteristic analysis of (a) 5 vol. \%, (b) 10 vol. \% MWCNT filled alumina composites and (c) Monel 400 alloy.

The fabricated MWCNT alumina composites are found to be brittle in nature [15]. So, the probability of cracks formation is quite high. Figure 5 represents the SEM images of cracks formation and propagation during machining of 5 and 10 vol. $\%$ MWCNT alumina composites. As alumina composites with 5 vol. \% MWCNTs being more brittle and of low electrical conductivity, longer span of micro cracks are observed. While, a comparatively shorter span of micro cracks are observed in samples with 10 vol. \% MWCNTs due to higher electrical conductivity. The appearance and formation of cracks disrupts the surface characteristics. Also, it has a more probability of breakage of the work piece, which is observed during machining of 5 vol. \% MWCNT filled alumina composites. Presence of sub surface micro cracks after the recast layers are also found on a closer analysis of the machined sample (Fig. 6). This shows the high susceptibility of the material under the influence of rapid thermal expansion. Further, it is expected that the high brittleness of the material have contributed to the surface micro cracks and sub surface cracks formation.

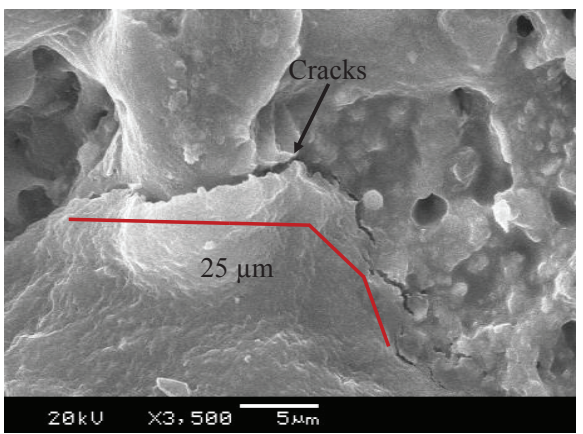

(a)

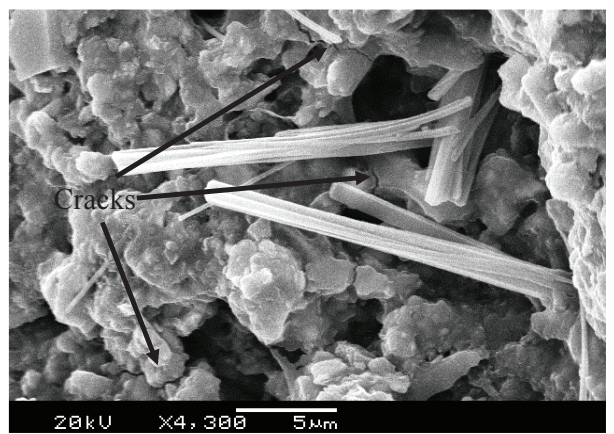

(b)

Figure 5. SEM micrograph for formation and propagation of cracks in (a) 5 vol. $\%$ and (b) 10 vol. \% MWCNT filled alumina composites.

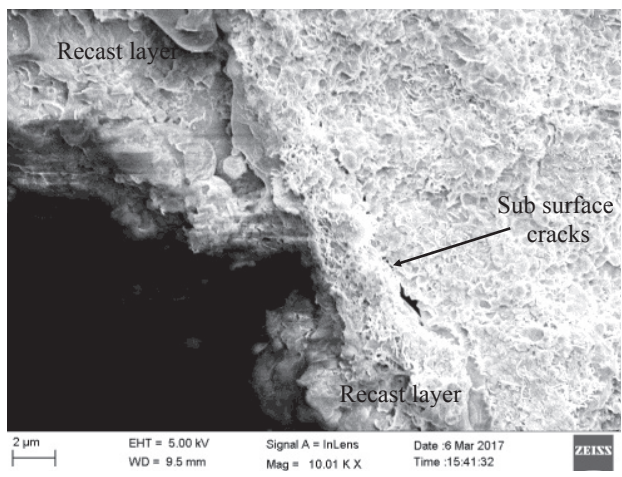

Figure 6. Sub surface analysis of 10 vol. \% MWCNT filled alumina composites. 


\section{Conclusions}

A WEDM process has been performed to machine MWCNT filled alumina composites. Two concentrations viz., 5 and 10 vol. \% MWCNTs are reinforced as the conducting phase in alumina. A more stable machining phenomenon is obtained with 10 vol. \% MWCNT filled alumina composites. The machining process of 5 vol. \% MWCNT filled alumina composites was found to be difficult owing to its low electrical conductivity. Some wire lag phenomena was also observed during the course of machining. The tendency of formation and occurrence of cracks are found to be more prominent at lower CNT concentration. A comparison of the alumina composites has been made with that of Monel 400 alloy with regards to machinability characteristics. Unlike metals where melting and evaporation is the main material removal mechanism, spalling effect was found to be more dominating for the case of ceramic composites.

\section{References}

1. N. Mohri, Y. Fukuzawa, T. Tani, N. Saito, K. Furutani, CIRP Ann - Manuf Techn, 45(1) (1996)

2. Y. Guo, G. Dang, J. Bai, Z. Lu, Key Eng Mater, 375-376 (2008)

3. N. Mohri, Y. Fukuzawa, T. Tani, T. Sata, CIRP Ann - Manuf Techn, 51(1) (2002)

4. A. Sabur, M. Y. Ali, M. A. Maleque, A. A. Khan, Procedia Eng, 56 (2013)

5. J Kozak, K. P. Rajurkar, N. Chandarana, J Mater Process Tech, 149 (2004)

6. Y. Guo, P. Hou, D. Shao, Z. Li, L. Wang, L. Tang, Mater and Manuf Process, 29 (2014)

7. C. Martin, B. Cales, P. Vivier, P. Mathieu, Mat Sci Eng A - Struct, 109 (1989)

8. T. Matsuo, E. Oshima, CIRP Ann - Manuf Techn, 41(1) (1992)

9. E. Ferraris, D. Reynaerts, B. Lauwers, CIRP Ann - Manuf Techn, 60 (2011)

10. R. Landfried, F. Kern, R. Gadow, Int J Appl Ceram Tec, 10(3) (2013)

11. K. M. Patel, P. M. Pandey, P. V. Rao, Int J Refract Met H, 27(5) (2009)

12. O. Malek, J. G. Julian, J. Vleugelss, W. Vanderauwera, B. Lauwers, M. Belmonte, Mater Today, 14(16) (2011)

13. L. Melk, M. L. Antti, M. Anglada, Ceram Int, 42(5) (2016)

14. M. A. Singh, D. K. Sarma, O. Hanzel, J. Sedlacek, P. Sajgalik, J Eur Ceram Soc, (2017)

15. O. Hanzel, J. Sedlacek, P. Sajgalik, J Eur Ceram Soc, 34 (2014) 